\title{
Thermal conductivity modeling using machine learning potentials: application to crystalline and amorphous silicon
}

\author{
X. Qian ${ }^{\text {a }}$, S. Peng ${ }^{\text {b }}$, X. Li ${ }^{c}$, Y. Wei ${ }^{b}$, R. Yang ${ }^{\text {a, * }}$ \\ a Department of Mechanical Engineering, University of Colorado, Boulder, CO, 80309, USA \\ ${ }^{\mathrm{b}}$ The State Key Laboratory of Nonlinear Mechanics (LNM), Institute of Mechanics, Chinese Academy of Sciences, Beijing, 100190, PR China \\ ' State Key Laboratory of Coal Combustion, School of Energy and Power Engineering, Huazhong University of Science and Technology, Wuhan, 430074, \\ Hubei, China
}

\section{A R T I C L E I N F O}

\section{Article history:}

Received 4 August 2019

Received in revised form

9 September 2019

Accepted 18 September 2019

Available online 26 September 2019

\section{Keywords:}

Thermal conductivity

Machine learning

Molecular dynamics

Phonons

\begin{abstract}
A B S T R A C T
First principles-based modeling on phonon dynamics and transport using density functional theory and the Boltzmann transport equation has proven powerful in predicting thermal conductivity of crystalline materials, but it remains unfeasible for modeling complex crystals and disordered solids due to the prohibitive computational cost to capture the disordered structure, especially when the quasiparticle 'phonon' model breaks down. Recently, machine learning regression algorithms show great promises for building high-accuracy potential fields for atomistic modeling with length scales and timescales far beyond those achievable by first principles calculations. In this work, using both crystalline and amorphous silicon as examples, we develop machine learning-based potential fields for predicting thermal conductivity. The machine learning-based interatomic potential is derived from density functional theory calculations by stochastically sampling the potential energy surface in the configurational space. The thermal conductivities of both amorphous and crystalline silicon are then calculated using equilibrium molecular dynamics, which agree well with experimental measurements. This work documents the procedure for training the machine learning-based potentials for modeling thermal conductivity and demonstrates that machine learning-based potential can be a promising tool for modeling thermal conductivity of both crystalline and amorphous materials with strong disorder.
\end{abstract}

() 2019 Elsevier Ltd. All rights reserved.
In the past decade, first principles-based calculations have become a powerful tool for predicting thermal conductivity of a wide range of bulk [1-6] and low-dimensional crystals [7-18]. Despite the successful application in modeling the phonon properties and thermal conductivity of simple crystals, first principles calculation for complex crystals and disordered materials remains challenging because the computational cost increases dramatically with the lowering of crystalline symmetry and the increasing size of unit cells $[19,20]$. It also becomes questionable to apply the quasiparticle 'phonon' picture assumed by the Boltzmann transport theory to complex crystals and disordered materials because a considerable amount of vibrational modes in these low-symmetry systems becomes diffusive or localized [21,22]. For modeling disordered systems, molecular dynamics (MD) simulations become a great choice because MD can easily incorporate detailed atomic

\footnotetext{
* Corresponding author.

E-mail address: ronggui.yang@colorado.edu (R. Yang).
}

structures including defects and local strains. However, MD has limited fidelity and accuracy owing to the lack of accurate interatomic potentials. Improving the accuracy of empirical interatomic potential is difficult because the $a b$ initio potential energy surface (PES) can hardly be fitted by simple functional forms that are artificially assigned based on the preknowledge of the interatomic bonding $[19,20,23,24]$. Using "rigid" or "definite" functional forms also severely limits the transferability among different atomic structures and material phases because it usually requires reformulating the fitting functionals.

Recently, machine learning potential (MLP) is emerging as a promising tool for bridging the gap between the first principles calculations and MD simulations for modeling thermal transport. Because MLP does not artificially assign functional forms, it does not suffer from the limited accuracy as empirical potential does while intrinsically incorporating anharmonic effects [25]. In the past five years, MLP has been successfully developed and used to model the structural, thermodynamic, and mechanical properties of some simple crystals such as $\mathrm{Si}, \mathrm{Ge}$, and GaN [26-28] and 
amorphous materials [29,30]. However, implementation of MLP for studying thermal transport has been rare and limited to simple crystals with relatively weak disorder such as vacancies and alloys [31,32]. In this work, we develop MLP for modeling thermal conductivity of both crystalline and amorphous materials, using silicon as an example. Although there exist quite a few methods of constructing MLP such as artificial neural network [26], supporting vector regression [33], and spectral neighbor analysis potential (SNAP), [34], the Gaussian approximation potential (GAP) [27,35] is chosen in this work because its accuracy of predicting interatomic forces is one of the highest among other MLP methods [36]. Furthermore, training of GAP models based on Gaussian process regression [37] only involves linear algebra without non-linear optimizations.

First, we briefly describe here the training of the GAP models for crystalline silicon (c-Si) and amorphous silicon (a-Si). To construct the training database, we use a stochastic method to generate random uncorrelated snapshots to sample the $a b$ initio PES. After GAP models are developed, thermal conductivity of both c-Si and a$\mathrm{Si}$ is calculated using equilibrium molecular dynamics (EMD) simulations. Fig. 1a shows the training strategy for building the GAP model for c-Si. Since our goal is to model thermal conductivity, the GAP model is required to fit and interpolate the PES around the equilibrium configuration accessible by thermal vibrations. Compared with ab initio molecular dynamics (AIMD) that sample a trajectory in the configurational space, a more efficient method is to stochastically generate uncorrelated snapshots with random displacements [38]:

$\boldsymbol{u}_{i}=\sum_{S} \boldsymbol{e}_{i s}\left\langle A_{i s}\right\rangle \sqrt{-2 \ln \zeta_{1}} \sin \left(2 \pi \zeta_{2}\right)$

where $\boldsymbol{u}_{i}$ is the displacement of the atom $i$ from the equilibrium position, $\boldsymbol{e}_{i s}$ and $\left\langle A_{i s}\right\rangle$ are the eigenvector and average amplitude of the atom $i$ participating in the vibration of the normal mode $s$, and $\zeta_{1}$ and $\zeta_{2}$ are two random numbers uniformly distributed in the interval of $(0,1)$. The amplitude $\left\langle A_{i s}(T)\right\rangle$ of the normal mode $s$ can be written as follows [38]:

$\left\langle A_{i s}\right\rangle=\sqrt{\frac{\hbar\left(n_{s}+\frac{1}{2}\right)}{m_{i} \omega_{s}}}$

where $m_{i}$ is the mass of the atom $i$ and $n_{s}$ is the Bose-Einstein distribution $n_{s}=\left[\exp \left(\frac{\hbar \omega_{s}}{k_{B} T}\right)-1\right]^{-1}$ at temperature $T$ and frequency $\omega_{s}$.

Clearly, generating displacement snapshots using Eqs. (1) and (2) requires the knowledge of force constants to obtain both normal mode frequency $\omega_{s}$ and eigenvectors $\boldsymbol{e}_{i s}$. For c-Si, $\omega_{s}$ and $\left\langle A_{i s}\right\rangle$ can be easily obtained from harmonic lattice dynamics calculations. Phonopy [39] package is used to generate supercells of cSi containing $5 \times 5 \times 5$ primitive cells with finite displacements; then, density functional theory (DFT) calculations are performed to obtain the interatomic forces using the projected augmented wave method implemented in the VASP package [40,41]. Exchangecorrelation energy is treated with the Perdew-Burke-Ernzerhof (PBE) functionals [42], and the plane-wave cutoff energy is set to $350 \mathrm{eV}, 40 \%$ higher than the maximum plane-wave energy recommended by the pseudopotential for Si $[43,44]$. After the vibrational frequencies and eigenvectors are obtained by solving the dynamic equation, the displacement amplitudes are calculated at $300 \mathrm{~K}$ and $600 \mathrm{~K}$, with 100 snapshots at each temperature. In total, 50000 local chemical environments (i.e., each atom with its neighbors in each snapshot) are sampled in the database. Selfconsistent field (SCF) calculations are then performed using the VASP package for each snapshot to obtain the energies and forces, which are then used as target observables to be fitted in the training data set. We note that thermal expansion is not included in this work because silicon has negligible thermal expansion below $600 \mathrm{~K}$
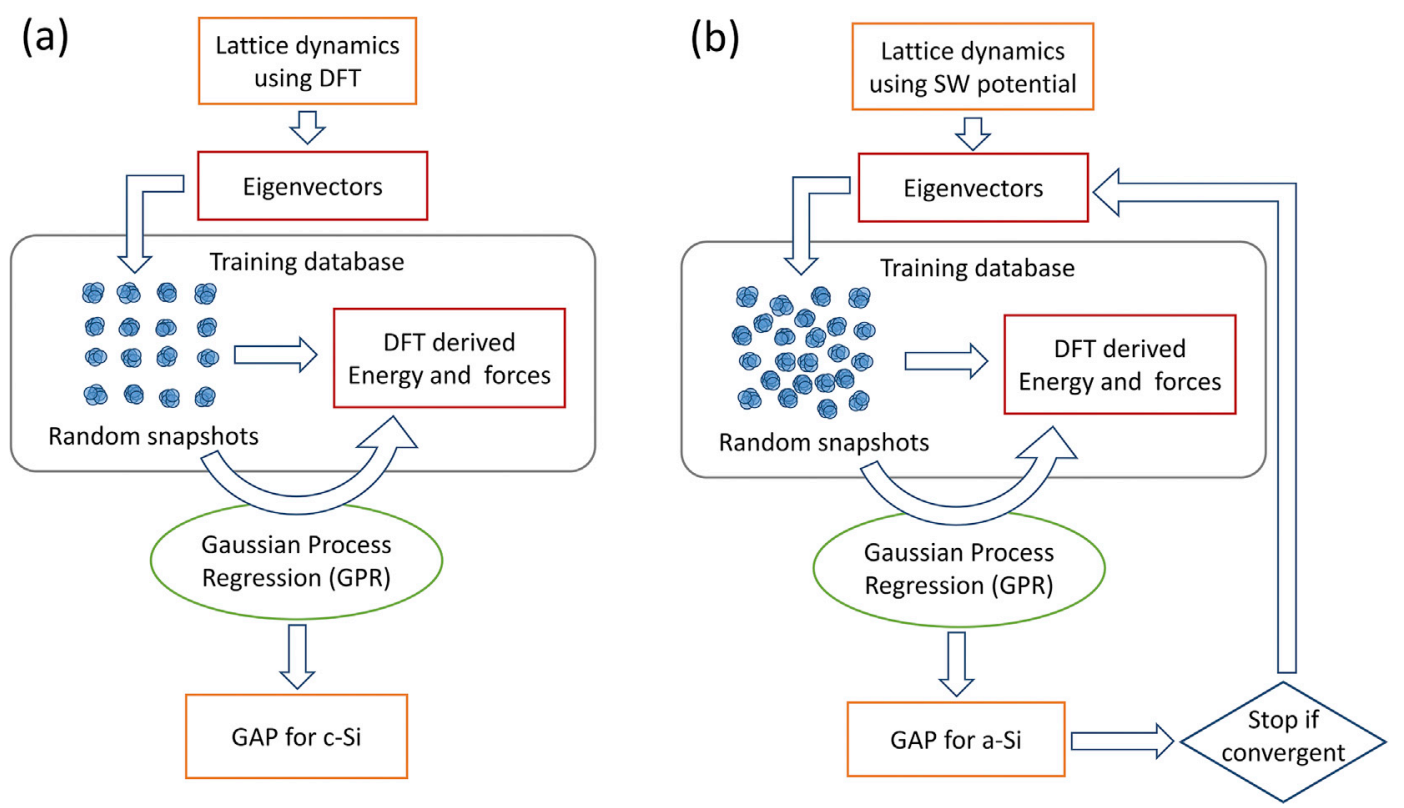

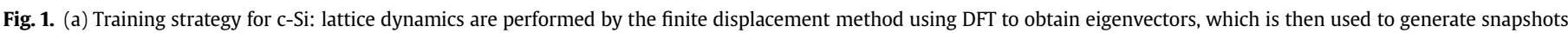

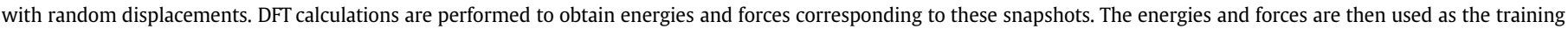

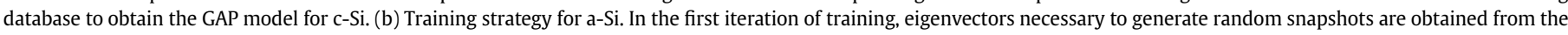

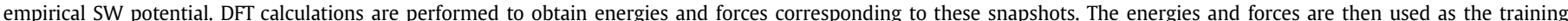

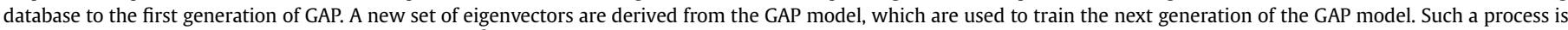
repeated until the energy change is less than $2 \times 10^{-3} \mathrm{eV} /$ atom. DFT, density functional theory; GAP, Gaussian approximation potential; SW, Stillinger-Weber. 
[45]. For strongly anharmonic materials, one could easily perform lattice dynamics with increased lattice constant to generate a new set of snapshots into the database [38] or directly perform AIMD simulations to construct the database [25]. In the training process, the energies and forces are expressed as a linear combination of a set of kernel functions specified in the smooth over atomic positions (SOAP) descriptor [46], and the associated linear coefficients are obtained through the sparsified Gaussian process regression formalism [47], the regression details of which can be found in the study by Szlachta et al [48]. Hyperparameters used for training are listed in Table 1, while the meaning of each parameter can be found in our previous work [25] and references [29,31]. Increasing the cutoff radius of neighboring atoms $r_{c u t}$ has negligible improvement on accuracy of phonon dispersion once it is beyond the second nearest neighbor ( $4.5 \AA$ ), which is also observed in fitting ab initio PES of silicon using either empirical functionals [49] or machine learning methods [28].

However, constructing the training database for a-Si is not as simple. First, it is non-trivial to obtain a relaxed amorphous network with atoms in equilibrium positions from DFT. Because a reasonable initial structure is important for the convergence of energy and forces when relaxing the atomic structures using DFT calculations, the classical MD simulation was performed first using Stillinger-Weber (SW) potential [50] to generate the initial structure of a-Si using a melt-quench method [30]. A c-Si simulation cell containing 216 atoms ( $3 \times 3 \times 3$ conventional cells) is first thermalized to $3000 \mathrm{~K}$ for $500 \mathrm{ps}$ using the Nose-Hoover reservoir (NPT ensemble) for the melting process. The temperature of the NoseHoover reservoir is then decreased to $1 \mathrm{~K}$ with a rate of $10 \mathrm{~K} / \mathrm{ps}$ and is kept at the final temperature to quench the system for another 2 ns. The final structure obtained from classical MD is then used as an initial guess for the a-Si network for performing geometry relaxation in DFT calculations. Although larger a-Si simulation cells can be obtained using this melt-quench method, the number of atoms is limited to $64-216$ atoms accessible by DFT calculations owing to the computational cost [51]. The simulation cell obtained using SW potential is then relaxed using the conjugated gradient algorithm implemented in the VASP package [41], until the atomic forces becomes weaker than $10^{-6} \mathrm{eV} / \AA$. The other challenge is the high computational cost to perform first principles lattice dynamics on an amorphous network containing hundreds of atoms. To mitigate this challenge, we use the training strategy for a-Si, as shown in Fig. 1b. Instead of generating random snapshots directly from DFT calculations, a set of trial eigenvectors is derived using the empirical SW potential. Because the optimized structure obtained from DFT calculations is not the same as the equilibrium structure obtained with the SW potential, there are soft vibrational modes with imaginary frequencies when performing lattice dynamics using SW potential. These soft modes are excluded when using Eq. (1) to sum the displacement over all modes for the first generation of random snapshots. After obtaining the trial snapshots, SCF calculations of the energy and forces were performed for each snapshot. The obtained forces and energies of the snapshots are recorded in the

Table 1

Hyperparameters for GAP with SOAP kernels.

\begin{tabular}{ll}
\hline$r_{\text {cut }}$ & $4.5 \AA$ \\
$d$ & $0.5 \AA$ \\
$\sigma_{v}$ for energy & $0.0001 \mathrm{eV} /$ atom; \\
$\sigma_{v}$ for forces & $0.001 \mathrm{eV} / \AA$ \\
$\sigma_{w}$ & $1.0 \mathrm{eV}$ \\
$\sigma_{a}$ & $0.5 \AA$ \\
$\zeta$ & 4 \\
$n_{\max }$ & 12 \\
$l_{\max }$ & 12 \\
\hline
\end{tabular}

GAP, Gaussian approximation potential. database for training the first generation of the GAP model. Note that the first generation of the generated random displacements does not correspond to the equilibrium population of the phonon modes. To minimize the possible error induced by the unphysical displacements, we adopted an iterative training process similar to the method used by Shulumba et al. [38] for developing the temperature-dependent effective potential method. The first generation of the GAP model is used to perform lattice dynamics again to generate a new set of snapshots for training the next generation of the GAP model. This process is repeated until the change of the total energy is less than $2 \times 10^{-3} \mathrm{eV} /$ atom and the soft modes disappear to ensure that the trained structure is dynamically stable using the GAP model. In this work, 50 snapshots are generated for training each generation of the GAP model, and convergence of atomic energy is achieved in the third iteration of training. The computational cost for training the potential itself is less, and one can perform the training within several processor hours (CPU hours) without any parallelization of the code. The major computational cost of generating GAP models instead comes from constructing the database. In this work, constructing the database of a-Si only involves 150 snapshots, which is comparable with the computational cost of computing force constants of simple crystals using the displacement method [38].

After the GAP models are trained, it is necessary to evaluate not only the accuracy of GAP models for reproducing the $a b$ initio energies and forces in the training database but also the accuracy in predicting energies and forces for snapshots that are not in the training database. The root-mean-square error (RMSE) of the energies and forces of GAP models are calculated by comparing with the data in the training databases. As shown in Fig. 2a and b, the RMSE of GAP reproducing the energy and interatomic forces in c-Si is $0.00057 \mathrm{eV} /$ atom and $0.0215 \mathrm{eV} / \mathrm{A}$, respectively. Compared with the RSME of interatomic forces $(0.29 \mathrm{eV} / \AA)$ using empirical SW potential, the RMSE of forces using the GAP model is one order of magnitude lower. As clearly shown in Fig. 2b, the SW potential has a steeper correlation to DFT forces than GAP models, which means that SW systematically overestimated the interatomic forces. Compared with our previous work using AIMD simulations for sampling the PES for crystalline $\mathrm{Zr}$ [25], a similar level of regression accuracy is achieved while the required number of snapshots is one order of magnitude lower. AIMD snapshots sample a trajectory on the $a b$ initio PES, which are intercorrelated. The stochastically generated snapshots are independent of each other, which results in a more effective sampling in the configurational space. To evaluate the accuracy in predicting forces of snapshots outside the training database, another set of snapshots was generated as the testing data set, and the corresponding forces and energies are calculated, as shown in Fig. 2a and b. The RMSE of energy and interatomic forces evaluated based on the testing data set is $0.00058 \mathrm{eV} /$ atom and $0.0217 \mathrm{eV} / \AA$, respectively, which is very close to the accuracy evaluated based on the training data set. To evaluate the accuracy of harmonic force constants, we calculated dispersion along high-symmetry paths and phonon frequencies at symmetryirreducible points in the first Brillouin zone. The harmonic force constants of GAP models are calculated using the finite displacement method [52], where symmetry-irreducible displacements of $0.03 \AA$ are imposed, and the corresponding interatomic forces here are calculated using the trained GAP model. As shown in Fig. 2c, phonon dispersion for c-Si using GAP is in excellent agreement with DFT calculation based on the PBE functional. In Fig. 2d, the phonon frequencies of symmetry-irreducible q-points are also calculated and compared using a q-mesh of $13 \times 13 \times 13$. The maximum deviation of phonon frequencies by GAP is $0.28 \mathrm{THz}$ compared with that obtained by PBE, indicating the GAP model accurately reproduces harmonic force constants. To evaluate how 

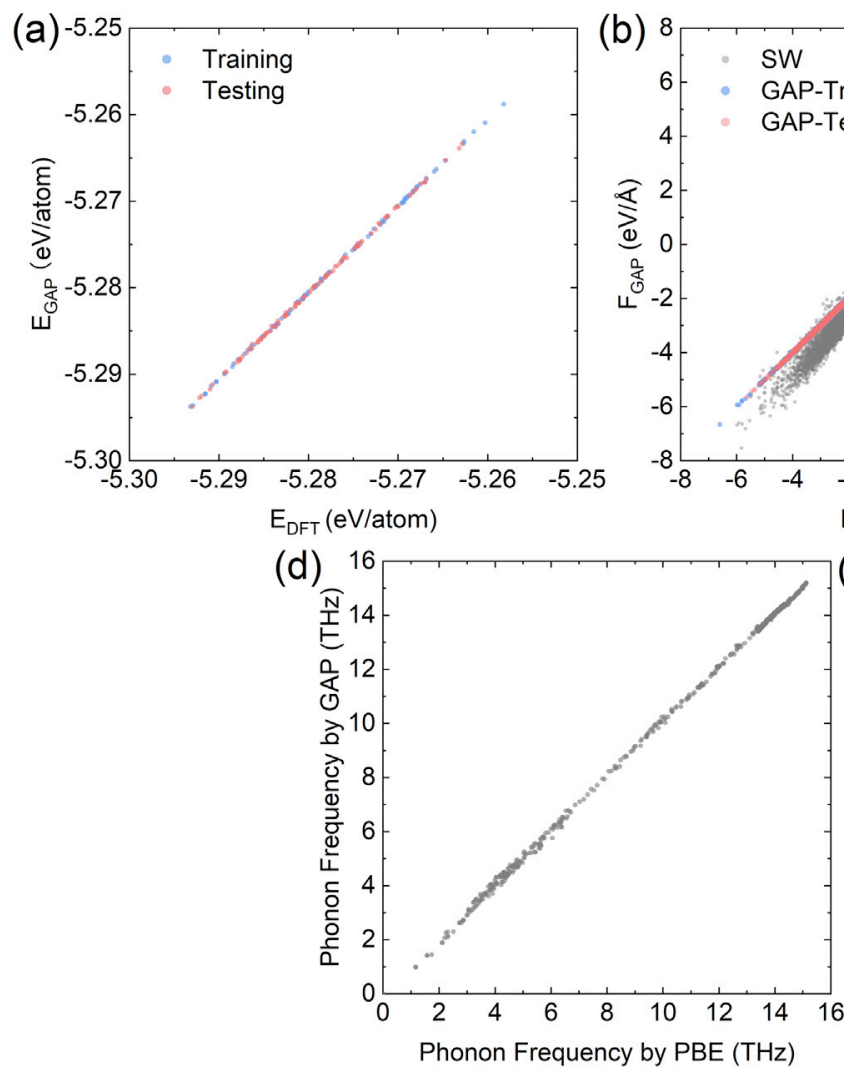
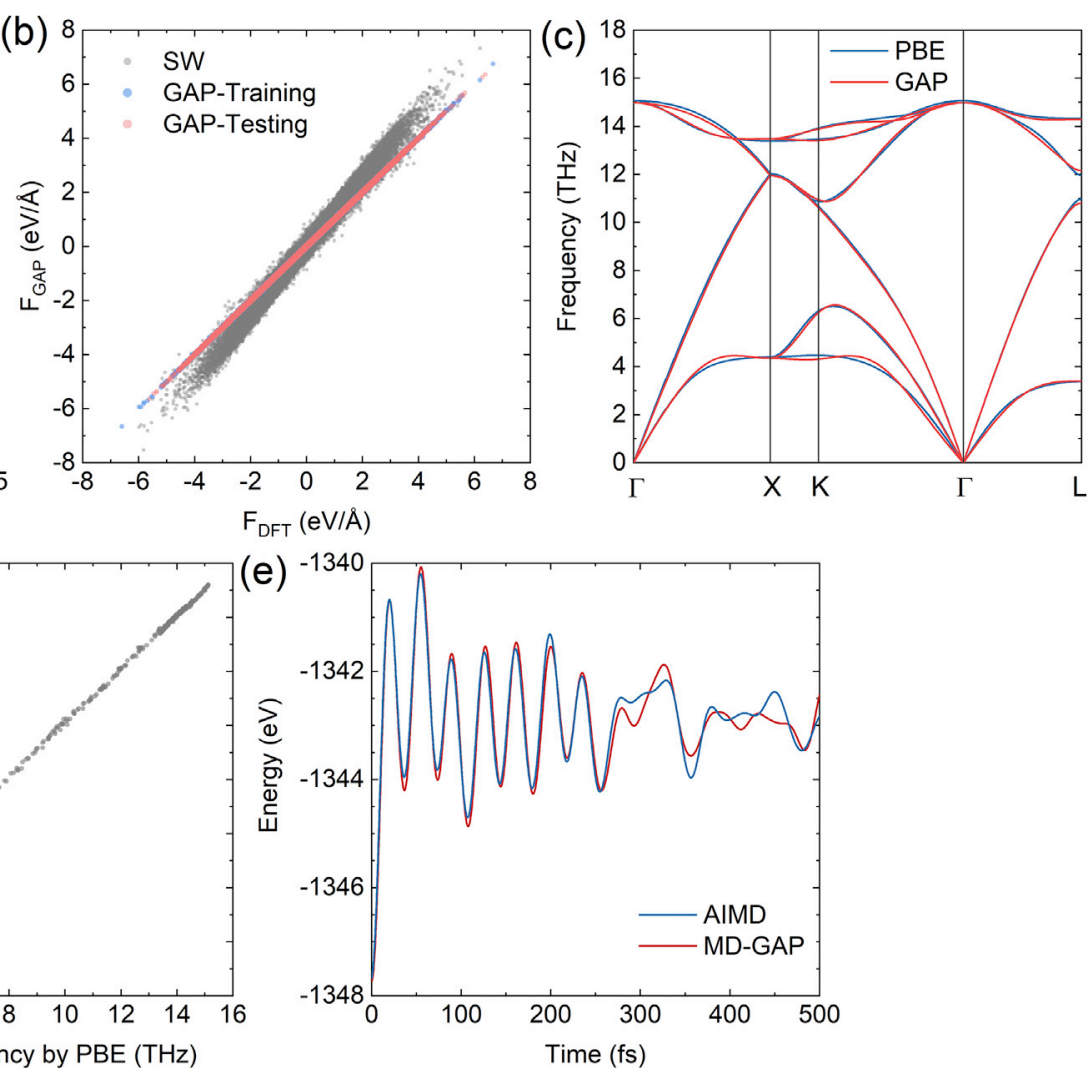

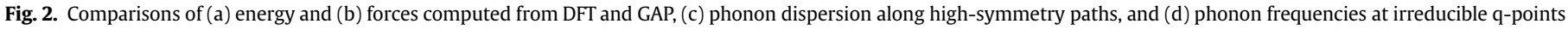

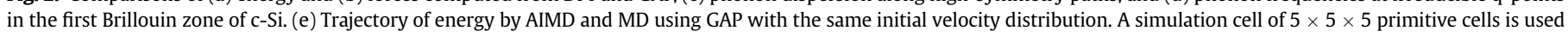

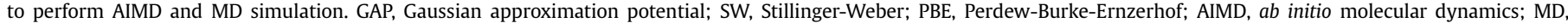
molecular dynamics; DFT, density functional theory.

the uncertainty for predicting energy accumulates over time, we performed both MD simulation with GAP and AIMD simulation using the same initial velocity distribution under microcanonical (NVE) ensemble, and the trajectory of potential energy fluctuation is recorded over time, as shown in Fig. 2e. In the first $250 \mathrm{fs}$, the energy fluctuation curve of MD using GAP follows closely with AIMD simulation. The two trajectories deviate significantly after 250 fs owing to the accumulation of errors in predicting energy and forces. Although any small amount of error in predicting forces and energy would eventually lead to such deviation of the trajectories owing to the chaotic nature of a many-body system [53], evaluating the maximum time before the energy fluctuation curve of MLP bifurcates from the trajectory of AIMD still serves as a validation of the fitting accuracy of MLP.

Fig. $3 \mathrm{a}$ and $\mathrm{b}$ show regression accuracy and prediction accuracy of energy and forces of a-Si. In the training database, the RMSE for reproducing energy and forces $(0.034 \mathrm{eV} /$ atom and $0.34 \mathrm{eV} / \AA$, respectively) is one order of magnitude higher that the c-Si owing to the much more complicated atomic structure and local atomic environments [29]. The accuracy in predicting energy and forces becomes lower when using the training data set. The RMSE of energy and forces is $0.066 \mathrm{eV} /$ atom and $0.54 \mathrm{eV} / \AA$, respectively, evaluated using the testing database. Similar to the case of $\mathrm{c}-\mathrm{Si}$, the accuracy of calculating interatomic forces using GAP models still outperforms the empirical SW potential by an order of magnitude, whose RMSE for the forces is as high as $1.5 \mathrm{eV} / \mathrm{A}$, similar to the case in c-Si. To further assess whether GAP could accurately capture the structural features of a-Si network, the radial distribution function (RDF) $g(r)$ of the equilibrium a-Si structures obtained from GAP and PBE is calculated and compared, as shown in Fig. 3c. It is observed that GAP can reasonably reproduce the RDF compared with PBE functionals, while SW potential falsely predicts a peak of RDF near $3 \AA$.

After the training process, GAP models are developed to predict interatomic forces needed for thermal conductivity calculations. EMD is performed to obtain thermal conductivity using the LAMMPS package [54]. First, the isothermal-isobaric ensemble (NPT) is used to thermalize the simulation cells for $400 \mathrm{ps}$ with a time step of 0.5 fs for both $\mathrm{c}-\mathrm{Si}$ and a-Si. The simulations are then switched to the microcanonical (NVE) ensemble for thermal conductivity calculation. In GAP models, heat flux $\mathcal{J}$ is expressed as follows:

$\mathcal{J}=\frac{1}{V} \sum_{i}\left(E_{i} \boldsymbol{v}_{i}-\boldsymbol{S}_{i} \cdot \boldsymbol{v}_{i}\right)$

where $V$ is the volume of the simulation cell, $E_{i}$ and $\boldsymbol{v}_{i}$ are the energy and velocity of the atom $i$, and the atomic virial stress tensor $\boldsymbol{S}_{i}$ is written as the outer product of relative position $\boldsymbol{r}_{j}-\boldsymbol{r}_{i}$ and local potential derivative with respect to the neighboring atom $\frac{\partial E_{i}}{\partial \boldsymbol{r}_{j}}$ :

$\boldsymbol{S}_{i}=\sum_{j}\left(\boldsymbol{r}_{j}-\boldsymbol{r}_{i}\right) \otimes \frac{\partial E_{i}}{\partial \boldsymbol{r}_{j}}$

Thermal conductivity is then calculated using the Green-Kubo formula:

$k=\frac{V}{3 k_{B} T^{2}} \int\langle\mathcal{J}(0) \cdot \mathcal{J}(t)\rangle d t$ 

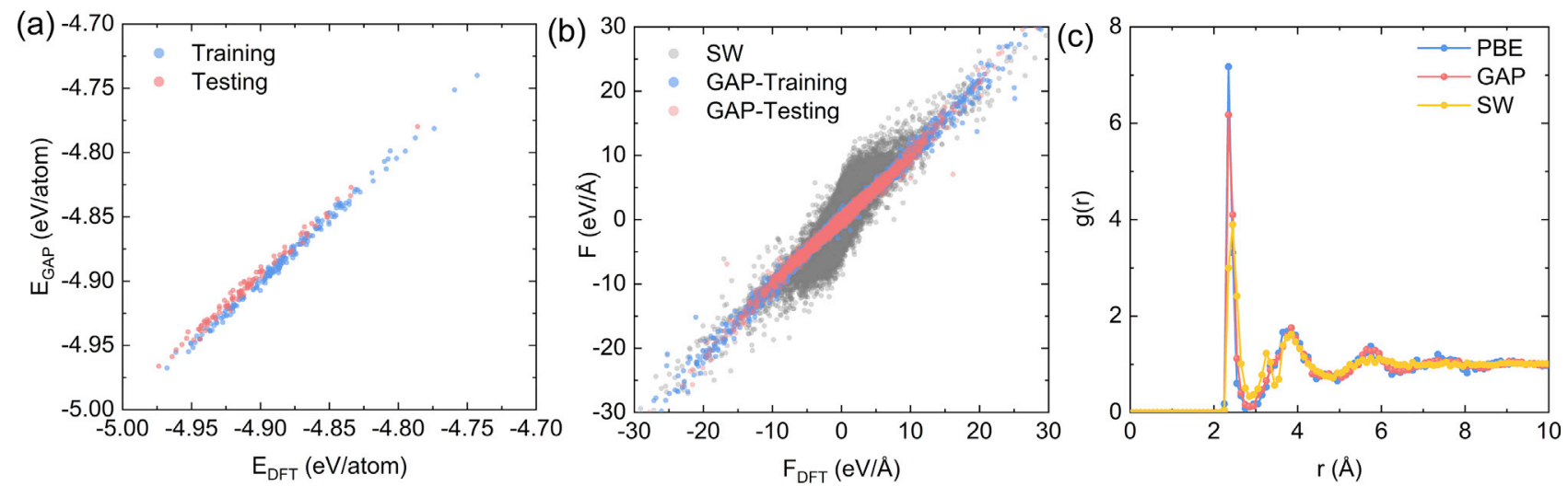

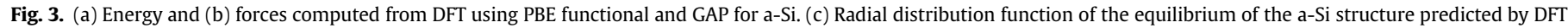

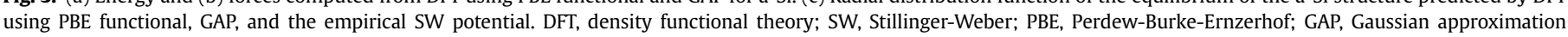
potential.

To perform the Green-Kubo integration, heat autocorrelation function $\langle\mathcal{J}(0) \cdot \mathcal{J}(t)\rangle$ is sampled every 5 fs and integrated up to 200 ps for c-Si and 40 ps for a-Si until the thermal conductivity values stopped increasing with the increase of the correlation time, as shown in Fig. 4a and b. To suppress the uncertainty, ten individual simulations with different initial velocity distributions are performed to average the heat autocorrelation function. At room temperature, the thermal conductivity values of c-Si and a-Si are found to be $121 \mathrm{~W} / \mathrm{mK}$ and $1.4 \mathrm{~W} / \mathrm{mK}$, respectively. In Fig. 4c, the thermal conductivity of c-Si obtained from EMD is compared with the values calculated by iteratively solving the Boltzmann transport equation (BTE), using the harmonic and third-order force constants
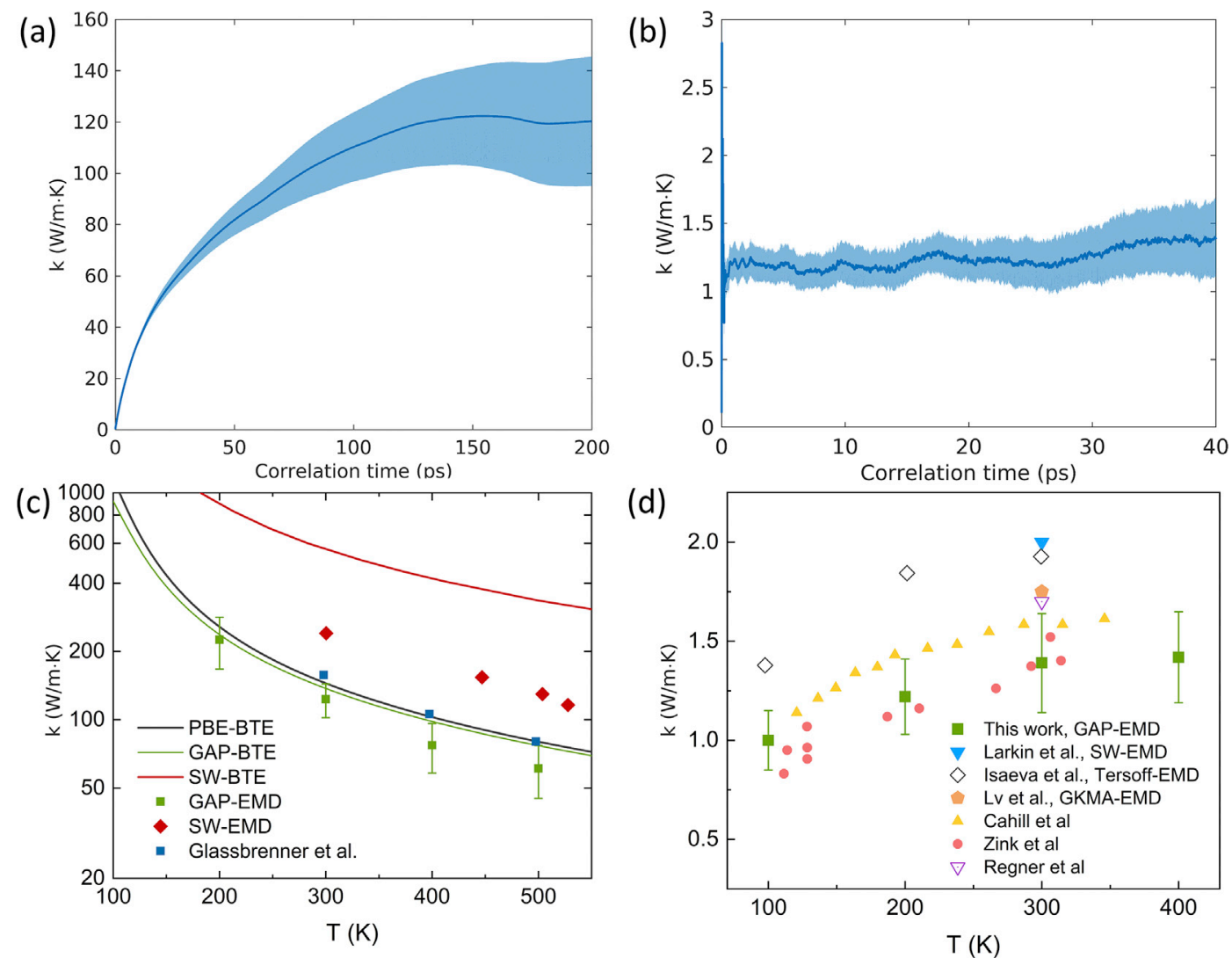

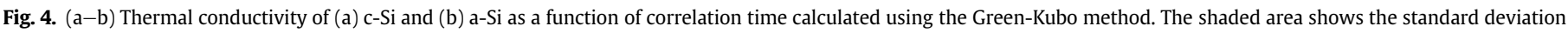

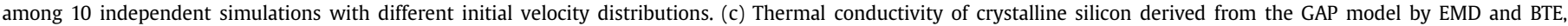

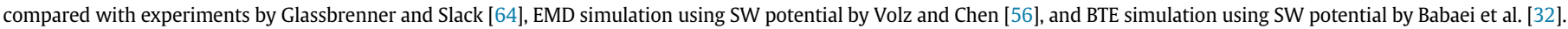

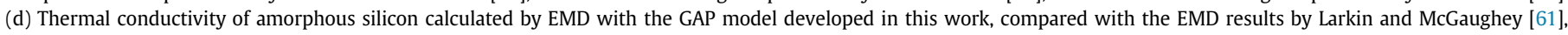

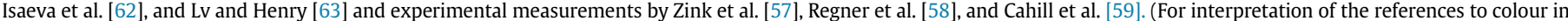

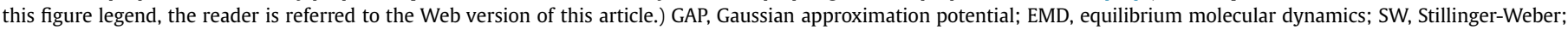
PBE, Perdew-Burke-Ernzerhof; GKMA, Green-Kubo model analysis; BTE, Boltzmann transport equation. 
obtained from PBE functionals $(145 \mathrm{~W} / \mathrm{mK})$ and the trained GAP model $(137 \mathrm{~W} / \mathrm{mK})$. Third-order anharmonic force constants of both the GAP model and PBE are calculated using the finite displacement method [52], using a supercell of $5 \times 5 \times 5$ primitive cells and a cutoff to the fourth nearest neighbor. The ShengBTE package [43] is used to iteratively solve the BTE with a $13 \times 13 \times 13$ $q$-mesh to sample the reciprocal $\boldsymbol{q}$ space. The slightly lower thermal conductivity obtained by solving BTE from GAP could be attributed to the error in predicting the interatomic forces. Even with the same GAP model, the thermal conductivity predicted by EMD simulation is $12 \%$ lower than that by solving BTE. At a high temperature of $500 \mathrm{~K}$, the difference between thermal conductivity predicted by $\operatorname{EMD}(61 \mathrm{~W} / \mathrm{mK})$ and BTE $(77 \mathrm{~W} / \mathrm{mK})$ increased to $\sim 20 \%$. There could be two reasons leading to the lower thermal conductivity from EMD simulations: (1) the classical statistics in MD could lead to overestimated scattering rates [55] and (2) MD simulations with GAP naturally include higher order anharmonicities, whereas the BTE approach truncates the anharmonic force constants to the third order. One possible reason that GAP overestimates anharmonicity could be the built-in variance nature of Gaussian process regression. It is known that the variance of predicting forces and energies increases as the input configuration moves close to the boundary of the sampled region, and the accuracy would decrease dramatically if one tires to extrapolate the PES to the unsampled region. In this work, the PES is sampled with an amplitude of thermal vibrations up to $600 \mathrm{~K}$. At higher temperatures close to $600 \mathrm{~K}$, the trained GAP would more frequently interpolate the PES in the region with larger variances in forces and energies, which could lead to overestimated anharmonicity. Such underestimated thermal conductivity by EMD using machine learning-based interatomic potential is also observed in transition metal dichalcogenide alloys [31]. Compared with the empirical SW potential [56], the GAP model still shows much higher accuracy in predicting thermal conductivity of c-Si. Fig. 4d shows thermal conductivity of a-Si obtained from EMD using the GAP for a-Si. The predicted thermal conductivity is $\sim 1.4 \mathrm{~W} / \mathrm{mK}$ at room temperature, within the range of measurement values of a-Si $(1-2 \mathrm{~W} / \mathrm{mK})$ [57-59]. Considering the fact that experimentally prepared a-Si usually contains different concentrations of hydrogen which reduces phonon localization and leads to higher thermal conductivity [60], the thermal conductivity obtained in this work could serve as an estimation for nonhydrogenated a-Si. On the other hand, the GAP model predicts a lower thermal conductivity for a-Si than that using the empirical potentials [61-63], probably due to the fact that the empirical potentials predict higher bonding stiffness than DFT calculation using PBE functionals, consistent with the trend we observed in cSi.

Finally, we briefly compare the computational cost of AIMD and MD using GAP. For a simulation cell containing 250 atoms, each ionic step in AIMD takes 110 s of CPU time, using 48 processors with the Brillouin zone sampled at the Gamma point. MD simulations using GAP models, trained in this work, take around $0.1 \mathrm{~s}$ per time step with the same number of atoms and processors, which is about three orders of magnitude faster than AIMD. However, MD calculations using GAP is much slower than that using the empirical potential with simple functionals such as SW potential, which takes only $0.3 \mathrm{~ms}$ per time step under the same condition. Therefore, it still remains challenging to implement MLP to model thermal conductivity of materials with multiple elements because the computational cost would further increase with the increasing number of elements [35].

In summary, we have developed GAP models with regression accuracy of $0.02 \mathrm{eV} / \AA \AA$ and $0.3 \mathrm{eV} / \AA \AA$ for interatomic forces in crystalline and $\mathrm{a}-\mathrm{Si}$, respectively, showing one-order-of-magnitude improvement in both energy and forces compared with the empirical SW potential. Thermal conductivity of c-Si and a-Si at room temperature is calculated to be $121 \mathrm{~W} / \mathrm{mK}$ and $1.4 \mathrm{~W} / \mathrm{mK}$, respectively, using EMD, agreeing reasonably well with experiments and first principles calculations. This work shows that GAP can be a promising tool for modeling thermal conductivity of both crystalline and amorphous materials with strong disorder.

\section{Conflict of interest}

The authors declare no conflict of interest.

\section{Acknowledgments}

This work is supported by the NSF (grant no. 1512776). Density functional theory calculations and training of the GAP models are performed using the Summit supercomputer, which is supported by the NSF (awards: ACI-1532235 and ACI-1532236), University of Colorado Boulder, and Colorado State University. Molecular dynamics simulations are performed using the Yuan supercomputer supported by Supercomputing Center of Chinese Academy of Sciences.

\section{References}

[1] W. Li, N. Mingo, L. Lindsay, D.A. Broido, D.A. Stewart, N.A. Katcho, Phys. Rev. B 85 (19) (2012).

[2] A. Ward, D.A. Broido, D.A. Stewart, G. Deinzer, Phys. Rev. B 80 (12) (2009).

[3] K. Esfarjani, G. Chen, H.T. Stokes, Phys. Rev. B 84 (8) (2011).

[4] D.A. Broido, M. Malorny, G. Birner, N. Mingo, D.A. Stewart, Appl. Phys. Lett. 91 (23) (2007) 231922.

[5] A. Ward, D.A. Broido, Phys. Rev. B 81 (8) (2010).

[6] L. Lindsay, D.A. Broido, T.L. Reinecke, Phys. Rev. Lett. 109 (9) (2012): 095901.

[7] G. Fugallo, A. Cepellotti, L. Paulatto, M. Lazzeri, N. Marzari, F. Mauri, Nano Lett. 14 (11) (2014) 6109-6114.

[8] L. Lindsay, D.A. Broido, N. Mingo, Phys. Rev. B 82 (11) (2010).

[9] L. Lindsay, D.A. Broido, N. Mingo, Phys. Rev. B 83 (23) (2011).

[10] X. Gu, R. Yang, J. Appl. Phys. 117 (2) (2015): 025102.

[11] H. Xie, M. Hu, H. Bao, Appl. Phys. Lett. 104 (13) (2014) 131906.

[12] A. Jain, A.J. McGaughey, Sci. Rep. 5 (2015) 8501.

[13] G. Qin, Q.B. Yan, Z. Qin, S.Y. Yue, M. Hu, G. Su, Phys. Chem. Chem. Phys. 17 (7) (2015) 4854-4858.

[14] B. Sun, X. Gu, Q. Zeng, X. Huang, Y. Yan, Z. Liu, R. Yang, Y.K. Koh, Adv. Mater. 29 (3) (2017) 1603297

[15] J. Zhu, H. Park, J.-Y. Chen, X. Gu, H. Zhang, S. Karthikeyan, N. Wendel, S.A. Campbell, M. Dawber, X. Du, M. Li, J.-P. Wang, R. Yang, X. Wang, Adv. Electron. Mater 2 (5) (2016) 1600040.

[16] X. Gu, B. Li, R. Yang, J. Appl. Phys. 119 (8) (2016): 085106.

[17] X. Gu, R. Yang, Appl. Phys. Lett. 105 (13) (2014) 131903.

[18] X. Gu, R. Yang, Phys. Rev. B 94 (7) (2016).

[19] X. Qian, X. Gu, R. Yang, J. Phys. Chem. C (2015) 28300-28308.

[20] X. Qian, X. Gu, R. Yang, Nano Energy 41 (2017) 394-407.

[21] J. Yang, X. Qian, W. Pan, R. Yang, Z. Li, Y. Han, M. Zhao, M. Huang, C. Wan, Adv Mater. 31 (24) (2019) 1808222.

[22] H.R. Seyf, L. Yates, T.L. Bougher, S. Graham, B.A. Cola, T. Detchprohm, M.-H. Ji, J. Kim, R. Dupuis, W. Lv, A. Henry, npj Comput. Mater 3 (2017) 49.

[23] A. Rohskopf, H.R. Seyf, K. Gordiz, T. Tadano, A. Henry, npj Comput. Mater 3 (1) (2017).

[24] P.C. Howell, J. Chem. Phys. 137 (22) (2012) 224111.

[25] X. Qian, R. Yang, Phys. Rev. B 98 (22) (2018) 224108.

[26] J. Behler, M. Parrinello, Phys. Rev. Lett. 98 (14) (2007) 146401.

[27] A.P. Bartok, M.C. Payne, R. Kondor, G. Csanyi, Phys. Rev. Lett. 104 (13) (2010) 136403.

[28] A.P. Bartok, J. Kermode, N. Bernstein, G. Csanyi, Phys. Rev. X 8 (4) (2018).

[29] V.L. Deringer, G. Csányi, Phys. Rev. B 95 (9) (2017): 094203.

[30] V.L. Deringer, N. Bernstein, A.P. Bartok, M.J. Cliffe, R.N. Kerber, L.E. Marbella, C.P. Grey, S.R. Elliott, G. Csanyi, J. Phys. Chem. Lett. 9 (11) (2018) 2879-2885.

[31] X. Gu, C.Y. Zhao, Comput. Mater. Sci. 165 (2019) 74-81.

[32] H. Babaei, R. Guo, A. Hashemi, S. Lee, Phys. Rev. Mat. 3 (2019) 074603.

[33] R.M. Balabin, E.I. Lomakina, Phys. Chem. Chem. Phys. 13 (24) (2011) $11710-11718$.

[34] A.P. Thompson, L.P. Swiler, C.R. Trott, S.M. Foiles, G.J. Tucker, J. Comput. Phys. 285 (2015) 316-330.

[35] A.P. Bartók, G. Csányi, Int. J. Quantum Chem. 115 (16) (2015) 1051-1057.

[36] Y. Zuo, C. Chen, X. Li, Z. Deng, Y. Chen, J. Behler, G. Csányi, A.V. Shapeev, A.P. Thompson, k. Mitchell, A. Wood, S.P. Ong, arXiv (2019), 1906.08888.

[37] C.E. Rasmussen, C.K.I. Williams, Gaussian Processes for Machine Learning, MIT Press, 2006. 
[38] N. Shulumba, O. Hellman, A.J. Minnich, Phys. Rev. B 95 (1) (2017).

[39] A. Togo, I. Tanaka, Scr. Mater. 108 (2015) 1-5.

[40] G. Kresse, J. Furthmuller, Comput. Mater. Sci. 6 (1996) 15-50.

[41] G. Kresse, D. Joubert, Phys. Rev. B 59 (3) (1991) 1758.

[42] J.P. Perdew, K. Burke, M. Ernzerhof, Phys. Rev. Lett. 77 (1996) 3865.

[43] W. Li, J. Carrete, N.A. Katcho, N. Mingo, Comput. Phys. Commun. 185 (6) (2014) 1747-1758.

[44] D. Sholl, J.A. Steckel, Density Functional Theory: a Practical Introduction, John Wiley \& Sons, 2011.

[45] Y. Okada, Y. Tokumaru, J. Appl. Phys. 56 (1984) 314.

[46] A.P. Bartók, R. Kondor, G. Csányi, Phys. Rev. B 87 (18) (2013) 184115.

[47] J. Schreiter, D. Nguyen-Tuong, M. Toussaint, Neurocomputing 192 (2016) $29-37$.

[48] W.J. Szlachta, A.P. Bartók, G. Csányi, Phys. Rev. B 90 (10) (2014) 104108.

[49] T.J. Lenosky, B. Sadigh, E. Alonso, V.V. Bulatov, T.D.d. 1. Rubia, J. Kim, A.F. Voter J.D. Kress, Model Simul, Mater. Sci. Eng 8 (2000) 825-841.

[50] J. Zi, K. Zhang, X. Xie, Phys. Rev. B 41 (18) (1990) 12915-12918.
[51] A. Pedersen, L. Pizzagalli, H. Jónsson, New J. Phys. 19 (6) (2017): 063018.

[52] G. Kresse, J. Furthmuller, J. Hafner, Europhys. Lett. 32 (9) (1995) 729-734.

[53] R.D. Skeel, SIAM J. Sci. Comput. 31 (2) (2009) 1363-1378.

[54] S. Plimpton, J. Comput. Phys. 117 (1995) 1-19.

[55] M. Puligheddu, Y. Xia, M.K.Y. Chan, G. Galli, Phys. Rev. Mat. 3 (2019) 085401.

[56] S.G. Volz, G. Chen, Phys. Rev. B 61 (4) (2000) 2651.

[57] B.L. Zink, R. Pietri, F. Hellman, Phys. Rev. Lett. 96 (5) (2006): 055902.

[58] K.T. Regner, D.P. Sellan, Z. Su, C.H. Amon, A.J. McGaughey, J.A. Malen, Nat. Commun. 4 (2013) 1640.

[59] D.G. Cahill, M. Katiyar, J.R. Abelson, Phys. Rev. B Condens. Matter 50 (9) (1994) 6077-6081.

[60] M.C. Wingert, J. Zheng, S. Kwon, R. Chen, Semicond. Sci. Technol. 31 (11) (2016) 113003.

[61] J.M. Larkin, A.J.H. McGaughey, Phys. Rev. B 89 (2014) 144303.

[62] L. Isaeva, G. Barbalinardo, D. Donadio, S. Baroni, arXiv (2019), 1904.02255.

[63] W. Lv, A. Henry, New J. Phys. 18 (1) (2016): 013028.

[64] C.J. Glassbrenner, G.A. Slack, Phys. Rev. 134 (4A) (1964) A1058-A1069. 